\title{
Myoepithelial carcinoma (malignant myoepithelioma) of the parotid gland arising in a pleomorphic adenoma
}

\author{
W G McCluggage, W J Primrose, P G Toner
}

\begin{abstract}
A myoepithelial carcinoma, a rare malignant salivary gland neoplasm, arose in a pleomorphic adenoma of the parotid gland. The initial tumour was a pleomorphic adenoma with epithelial and myoepithelial elements. Subsequently the tumour recurred twice and was characterised by invasion of the mandible. Histological examination of the second recurrence showed a malignant spindle cell neoplasm with an infiltrative growth pattern and a high mitotic rate. There was involvement of local lymph nodes. The immunophenotype was characteristic of myoepithelial differentiation: tumour cells stained positively with anticytokeratin antibodies, S-100 protein, $\alpha$ smooth muscle actin, and vimentin. Electron microscopy confirmed myoepithelial differentiation, with small foci of keratinocytic phenotype. Large numbers of tumour cell nuclei were reactive with the anti-p53 antibody, DO-7, in contrast to the two previous resections. Thus malignant transformation of a pleomorphic adenoma may involve myoepithelial as well as epithelial elements. Accumulation of p53 protein, perhaps through mutational events, may have played a role in this malignant transformation.

(f Clin Pathol 1998;51:552-556)
\end{abstract}

Keywords: salivary gland; pleomorphic adenoma; myoepithelial carcinoma; $\mathrm{p} 53$ protein

Pleomorphic adenoma or mixed tumour is the most common neoplasm of salivary gland origin. Malignant transformation is relatively uncommon but is well described. ${ }^{1}$ The most common malignant tumour to arise in a pleomorphic adenoma is a carcinoma, usually undifferentiated carcinoma or adenocarcinoma, not otherwise specified. In this report we present an ultrastructural and detailed immunohistochemical analysis of a myoepithelial carcinoma (malignant myoepithelioma) which arose in a pleomorphic adenoma of the parotid gland.

\section{Case report}

A 55 year old man presented with a six month history of painless swelling in the region of the left parotid gland. On examination, a $6 \mathrm{~cm}$ diameter firm tumour mass was palpable in the gland. There was no evidence of facial nerve palsy. Computed tomography (CT) revealed a large tumour in the left parotid gland extending anteriorly onto the masseter muscle and overlying the sternocleidomastoid muscle posteriorly. There was no evidence of bony erosion or extension of tumour deep to the mandible. A left superficial parotidectomy was performed. Postoperatively the patient made a good recovery, with full facial nerve function.

Fifteen months later a recurrent mass developed in the same region. On examination, this was mainly situated in the left masseter muscle but a few satellite nodules were present in the region of the previous scar. CT confirmed the presence of recurrent tumour which involved the body and ramus of the mandible. $\mathrm{He}$ underwent a left radical parotidectomy and hemi-mandibulectomy with sacrifice of the facial nerve. Postoperatively he made a good recovery.

Five months later he developed a further mass in the region of the scar. This was resected. The patient received postoperative radiotherapy. There was no clinical or radiological evidence of recurrent tumour or of distant metastasis at a six month review.

\section{Methods}

The surgical specimens were fixed in formalin. Sections for histological examination were routinely processed in paraffin wax and stained with haematoxylin and eosin. Immunohistochemical staining was performed using a standard streptavidin-biotin-peroxidase method (Dako, Copenhagen, Denmark). The following monoclonal antibodies were used: AE1/AE3 (Dako), S-100 protein (Diagnostic Products, Abingdon, UK), $\alpha$ smooth muscle actin (Sigma, Poole, UK), desmin (Dako), glial fibrillary acidic protein (GFAP; Dako), vimentin (Dako), epithelial membrane antigen (EMA; Dako) and carcinoembryonic antigen (CEA; Dako). Sections were also stained with the anti-p53 monoclonal antibody DO-7 (Novocastra, Newcastleupon-Tyne, UK).

Transmission electron microscopy was performed on retained tissue from the second recurrence, which had been fixed in formalin but not processed in paraffin wax. Ultrathin sections were stained with uranyl acetate and lead citrate.

\section{Pathological findings}

The initial resection consisted of a well circumscribed tumour weighing $75 \mathrm{~g}$ and measuring $6 \mathrm{~cm}$ in maximum dimension.

Histological examination showed a pleomorphic adenoma with epithelial and myoepithelial elements. The epithelial element consisted of 

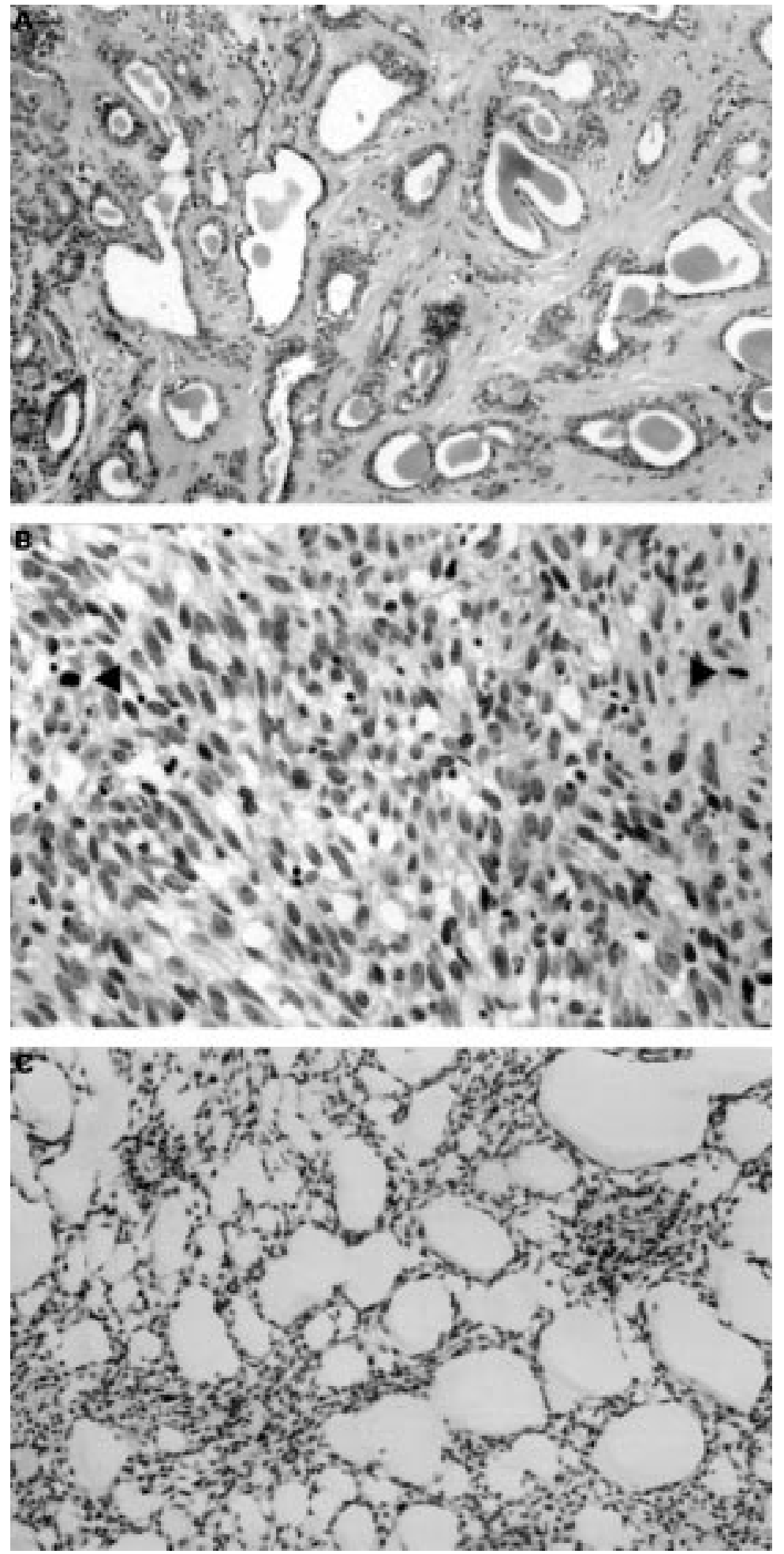

Figure 1 (A) Area of typical pleomorphic adenoma from the first resection, composed of ducts set in a hyalinised stroma. (B) Second recurrence showing a cellular spindle cell neoplasm. Several mitotic figures are identified (arrows). (C) Abundant myxoid stroma between tumours cells in the second recurrence, resulting in a "lace-like" pattern.

ducts and tubules set in a hyalinised stroma (fig 1A). Elsewhere, the tumour was composed of myoepithelial cells in a myxoid stroma. Within these areas, scattered ducts were present and there were foci of squamous differentiation. The myoepithelial areas were unusually cellular, but there was little nuclear pleomorphism and few mitotic figures. No vascular invasion or areas of necrosis were identified. The neoplasm was largely surrounded by a thin fibrous capsule, but in some areas small foci of tumour were seen outside the capsule. Tumour was present at the surgical resection margin.

The first resected recurrence consisted of a tumour measuring $5 \mathrm{~cm}$ in maximum dimension. Sectioning revealed solid and cystic areas. The tumour eroded the body and ramus of the mandible.

Histological examination showed that the tumour was composed predominantly of myoepithelial cells similar to those seen in the initial specimen. Epithelial elements consisting of ducts and tubules were focally present. Again, there was little nuclear pleomorphism and few mitotic figures. There was no necrosis or vascular invasion. In the mandible, the presence of tumour infiltrating between bony trabeculae was confirmed. The histological features were felt to represent recurrent pleomorphic adenoma but the infiltrative growth pattern and erosion of bone suggested low grade malignant transformation, involving myoepithelial elements. Tumour was present at the surgical resection margin.

The second resected recurrence consisted of a tumour mass measuring $7 \mathrm{~cm}$ in maximum dimension. Sectioning revealed solid and cystic areas.

Multiple sections were examined histologically. They showed a cellular tumour composed almost entirely of cells with ovoid to spindle shaped nuclei (fig 1B). There was a moderate amount of cytoplasm, which was either clear or eosinophilic. Small collections of epithelioid cells, which often showed keratinisation, were distributed diffusely throughout the sections examined. There was an abundant myxoid stroma between tumour cells which focally resulted in a "lace-like" pattern (fig 1C). There was moderate nuclear pleomorphism and mitotic figures were readily identified. A formal mitotic count revealed areas in which there were $10-20$ mitoses per 10 high power fields. The tumour infiltrated adipose tissue and skeletal muscle. Several lymph nodes present around the periphery of the neoplasm were infiltrated by tumour.

\section{Immunohistochemical findings}

Ducts and tubules in the first and second resections were immunoreactive for $A E 1 / A E 3$, EMA, and CEA but negative with the other antibodies. Myoepithelial cell areas stained focally positive for AE1/AE3, S-100 protein, $\alpha$ smooth muscle actin, desmin, and vimentin. Small numbers of myoepithelial cell nuclei $(<10 \%)$ were immunoreactive with DO-7.

Tumour cells in the second recurrence stained focally positive for AE1/AE3 (fig 2A), S-100 protein (fig $2 \mathrm{~B}$ ), $\alpha$ smooth muscle actin, desmin, and vimentin. Large numbers of tumour cell nuclei $(>50 \%$ ) were positive with DO-7 (fig 2C). Tumour cells were negative with the other antibodies.

\section{Electron microscopy}

Ultrastructural examination showed loosely interdigitating tumour $\mathrm{c}$ ells with focal adhesion 

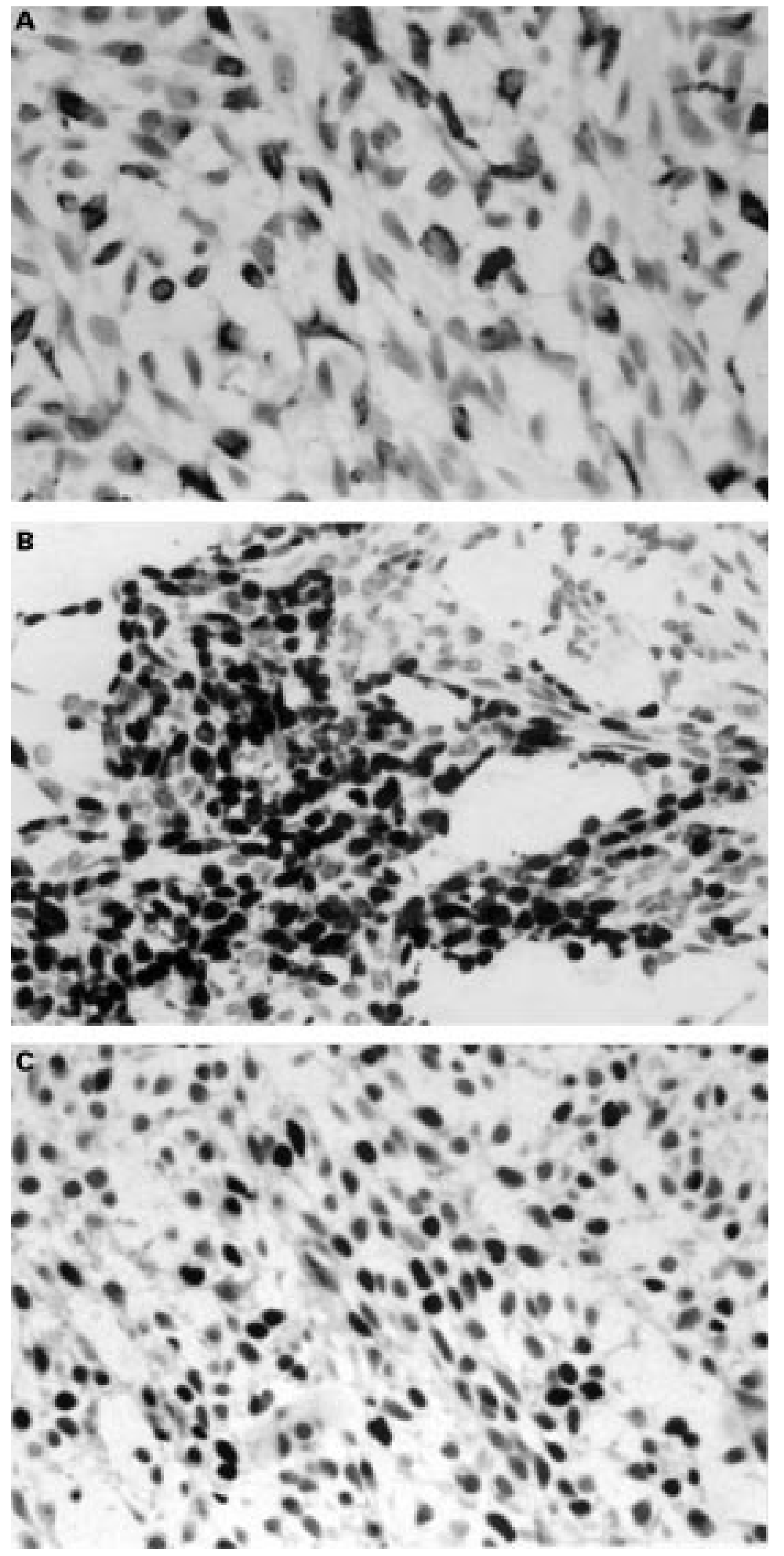

Figure 2 (A) Positive immunohistochemical staining of myoepithelial carcinoma with AE1/AE3. (B) Positive immunohistochemical staining of myoepithelial carcinoma with S-100 protein. (C) Second recurrence, showing myoepithelial carcinoma in which large numbers of tumour cell nuclei are positive with DO-7, an anti-p53 antibody.

specialisations, set in an abundant myxoid stroma containing small numbers of collagen fibrils. Tumour cells had ovoid to spindle shaped nuclei and a moderate amount of cytoplasm, with variable proportions of cell organelles. Many of the more elongated tumour cells contained bundles of thin cytoplasmic myofilaments with dense bodies. Subplasmale- mmal densities were present and the cells were often asymmetrically adherent to a prominent basal lamina (fig 3A). Such polarisation is typical of myoepithelial cells and contrasts with the more circumferential distribution seen in smooth muscle differentiation. Occasional groups of cells showed classical keratinocytic features, with prominent desmosomes linked to cytoplasmic bundles of dense intermediate filaments of cytokeratin type (fig 3B). Aggregates of intracytoplasmic desmosomes were occasionally encountered. These keratinocytic areas corresponded to the focal squamoid areas seen on light microscopy. These were clearly not entrapped ductal remnants, since they did not possess a basal lamina and merged imperceptibly with the surrounding spindle cells. A curious feature was the focal occurrence of tubular differentiation within keratinocytes, reminiscent of the pattern seen in sweat duct structures (fig 3C).

\section{Discussion}

Salivary gland neoplasms composed exclusively or predominantly of myoepithelial cells are relatively uncommon. Most behave in a benign fashion and are designated myoepithelioma. The malignant counterpart, myoepithelial carcinoma or malignant myoepithelioma, is rare but is one of several new entities included in the updated classification of salivary gland neoplasms by the World Health Organisation in $1991 .^{2}$ Myoepithelial carcinoma may occasionally develop in a pre-existing pleomorphic adenoma, but most often arises de novo. ${ }^{3}$ We have identified nine previously reported cases of myoepithelial carcinoma arising in a pleomorphic adenoma. ${ }^{4-7}$

Two criteria must be satisfied to establish a diagnosis of myoepithelial carcinoma: the neoplastic cells must show myoepithelial differentiation and the tumour must be malignant. In the present case, myoepithelial differentiation was established by immunohistochemistry and electron microscopy. The immunophenotype was characteristic: tumour cells were immunoreactive with antibodies to cytokeratin (AE1/ AE3), S-100 protein, and $\alpha$ smooth muscle actin. An unusual feature was the focal positivity with desmin, a marker of smooth muscle differentiation. Desmin positivity is not usual in myoepithelial cells and as far as we are aware has not previously been described in salivary gland myoepithelial tumours. The significance of desmin positivity in the present case is uncertain but we feel the immunohistochemical and ultrastructural findings clearly demonstrated myoepithelial differentiation. Electron microscopy showed typical myoepithelial cell features, along with evidence of focal keratinocytic differentiation. Establishing a diagnosis of malignancy was less straightforward. The infiltrative growth pattern with involvement of bony structures was suggestive of but was not in itself indicative of a malignant tumour, since these features are compatible with a benign pleomorphic adenoma. However, the combination of nodal involvement, nuclear pleomorphism, and mitotic activity was considered suf- 

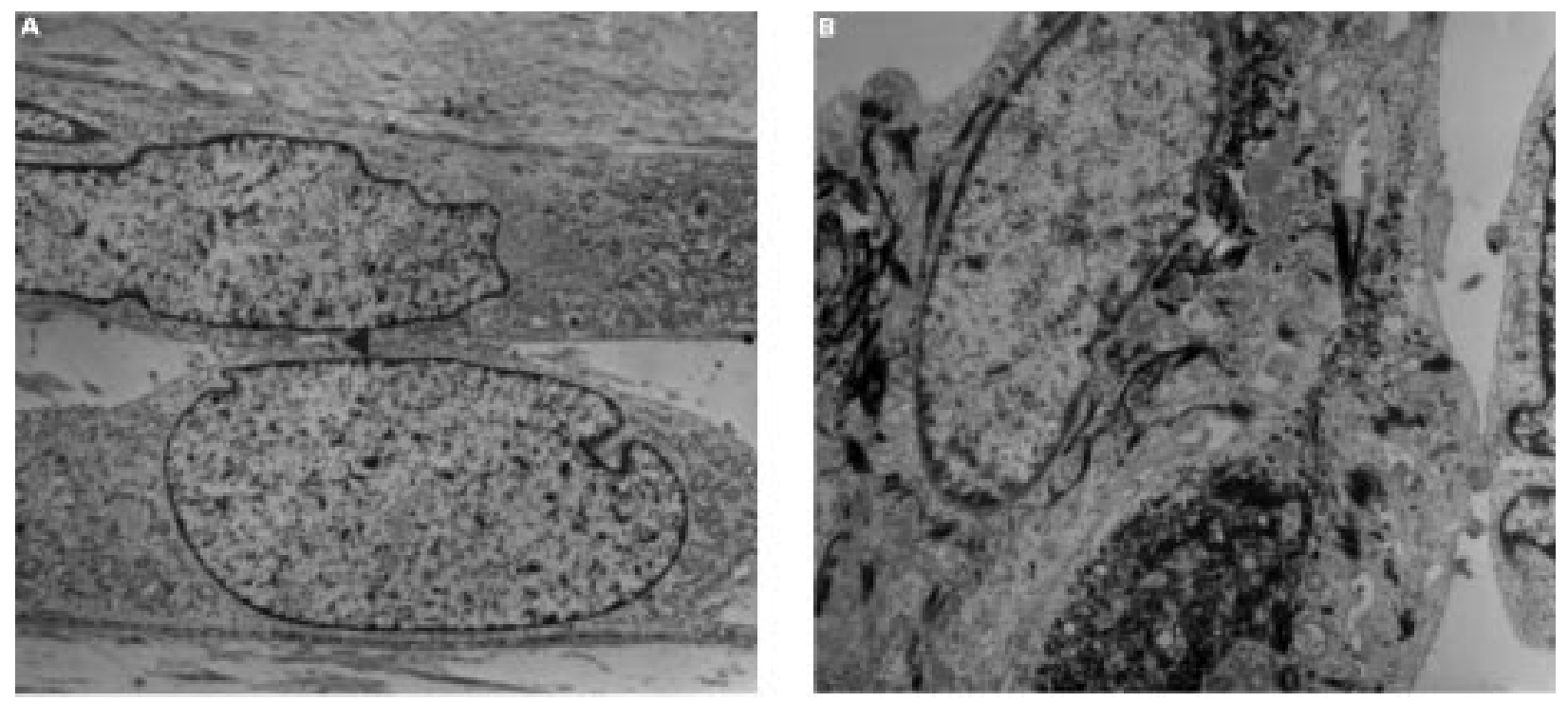

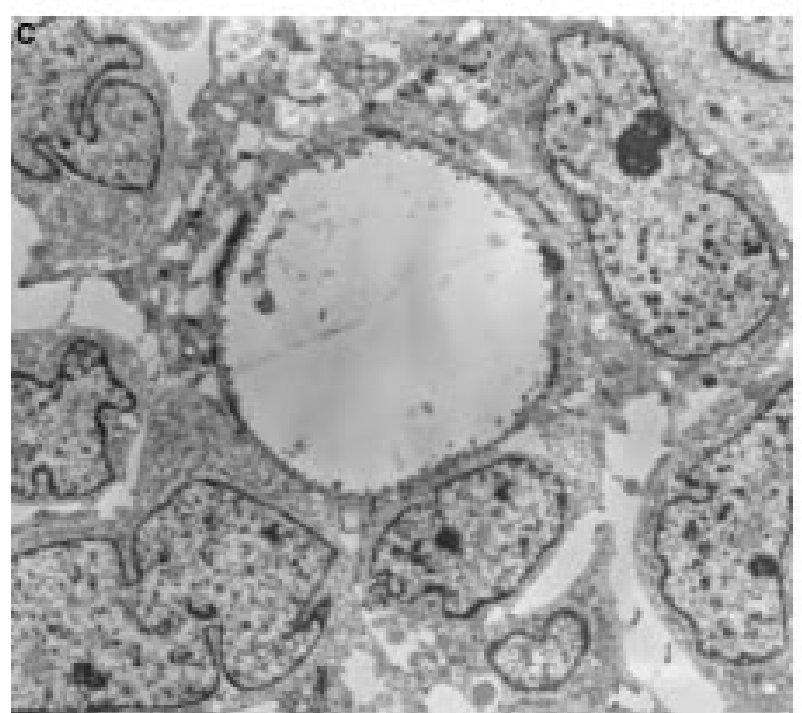

Figure 3 (A) Two adjacent tumour cells make contact at their "apical" surfaces, where a small desmosome is seen (arrow), and display a well formed basal lamina at the opposite side $(\times 5780)$. (B) In an area of typical keratinocytic differentiation, cells are attached by desmosomes, linked to dense tonofilament bundles. There is an aggregate of intracytoplasmic desmosomes, with associated tonofilaments $(\times 8720) .(C)$ $A$ ductal structure is present in a keratinocytic focus $(\times 3780)$. ficient to justify the designation of 43 years. In the case described by Singh and myoepithelial carcinoma.

In the present case, the malignancy clearly arose in a pleomorphic adenoma. In other cases, demonstration of a pre-existing adenoma may be more difficult, since the malignant tumour may overgrow and completely obliterate any residual benign elements. To establish that a myoepithelial carcinoma has arisen in a pleomorphic adenoma may require histological examination of the entire tumour in order to demonstrate a small residual focus of adenoma. In the absence of such histological evidence, a history of sudden rapid increase in the size of a mass which has been present for some time is suggestive of an origin in a benign adenoma. There was no histological evidence of a pre-existing adenoma in the second recurrence in our case, despite examination of multiple sections. There was also an unusually short time period between resection of the primary tumour and the development of recurrences. Di Palma and Guzzo ${ }^{4}$ described five cases of malignant myoepithelioma arising in a pleomorphic adenoma. Malignancy developed after variable time intervals, ranging from six to
Cawson, ${ }^{6}$ malignancy developed in a parotid gland mass which had been present for 15 years. In this case, there was histological evidence of a pre-existing pleomorphic adenoma.

Pleomorphic adenomas are well known for their tendency to recur locally following surgical resection. In both the initial resections, tumour was present at the surgical margin. In addition, the first two resections showed unusual histological features: initially the tumour had an unusually cellular myoepithelial component, and in the first recurrence the tumour was predominantly composed of cellular myoepithelial areas. The presence of tumour in the surgical resection margins and the unusual histology may indicate that a more aggressive approach was necessary in this patient. This shows that close cooperation between surgeon and histopathologist is mandatory as pathological features of this type may suggest the possibility of future malignant transformation.

A feature of the tumour in our case was the presence of small foci of squamoid differentia- 
tion with focal keratinisation. This was confirmed by ultrastructural examination, which also revealed focal tubular differentiation of keratinocytes, reminiscent of the pattern seen in sweat duct structures. Squamoid differentiation is one of the recognised manifestations of neoplastic myoepithelial cells and is acceptable as a minor component in myoepithelial carcinoma. ${ }^{3}$ In this case it was considered that the foci of squamoid differentiation represented an integral part of the tumour and not entrapped epithelial elements from the preexisting pleomorphic adenoma, since they were distributed diffusely throughout the neoplasm, merged imperceptibly with the surrounding myoepithelial cells, and showed no evidence of a residual basal lamina.

Most carcinomas arising from pleomorphic adenomas have the pattern of undifferentiated carcinoma or adenocarcinoma, not otherwise specified. ${ }^{13}$ Rarer types include mucoepidermoid carcinoma, squamous carcinoma, adenoid cystic carcinoma, acinic cell carcinoma, and clear cell adenocarcinoma. ${ }^{13}$ Occasionally a pleomorphic adenoma which is histologically benign may inexplicably metastasise. The term benign metastasising pleomorphic adenoma has been applied to such cases. Rarely, a carcinosarcoma or true malignant mixed tumour may develop, ${ }^{8}$ where both epithelial and stromal elements show malignant features. In such cases the sarcomatous component may predominate. In the present case, while the malignant spindle cell pattern initially suggested a sarcoma, immunohistochemistry was useful in confirming dual immunoreactivity with anticytokeratin and S-100 protein antibodies - this is characteristic of myoepithelial differentiation, features of which were also demonstrable by electron microscopy. It is important to consider a diagnosis of myoepithelial carcinoma when confronted with a malignant tumour arising in a pleomorphic adenoma. Most carcinomas arising in this situation are highly aggressive neoplasms. This may not be the case with myoepithelial carcinoma, which appears to be a low grade malignancy when arising in a pleomorphic adenoma, but to be more aggressive and to have a higher metastatic potential when arising de novo. ${ }^{4}$
Mutation of the p53 tumour suppressor gene, located on the short arm of chromosome 17 , is among the most commonly detected genetic abnormalities in human neoplasia. Wild-type p53 in normal tissues is generally not detectable by immunohistochemistry, owing to a combination of low expression and short half life. Mutation of the p53 gene results in a conformational change in the protein, which becomes stabilised, thus allowing for immunohistochemical detection. Previous studies have suggested that carcinomas arising in pleomorphic adenomas may be a result of genetic mutations, especially of the p53 gene. ${ }^{9}{ }^{10}$ In the present case, there was positive DO-7 staining of large numbers of tumour cell nuclei in the myoepithelial carcinoma. In the first and second resections, epithelial areas were negative, but there was focal staining of the cellular myoepithelial areas. This suggests that inactivation of p53 protein, perhaps through mutational events, may have played an important role in the development of myoepithelial malignancy in our case.

1 Gnepp DR. Malignant mixed tumors of the salivary glands: a review. Pathol Annu 1993;28:279-328.

2 Seifert G, Sobin LH. Histological typing of salivary gland tumours. World Health Organisation international histological classification of tumours, 2nd ed. New York: Springer-Verlag, classificat 1991.

3 Ellis GL, Auclair PL. Malignant epithelial tumors. In: Ellis GL, Auclair PL, eds. Atlas of tumor pathology. Tumors of the salivary glands, 3rd ed. Washington: AFIP, 1996:337-43.

4 Di Palma S, Guzzo M. Malignant myoepithelioma of salivary glands: clinicopathological features of ten cases. Virchows Arch (A) 1993;423:389-96.

5 Di Palma S, Pilotti S, Rilke F. Malignant myoepithelioma of parotid gland arising in a pleomorphic adenoma. Histopathology 1991;19:273-5.

6 Singh R, Cawson RA. Malignant myoepithelial carcinoma (myoepithelioma) arising in a pleomorphic adenoma of the parotid gland. An immunohistochemical study and review of the literature. Oral Surg Oral Med Oral Pathol of the literature.

7 Tortoledo ME, Luna MA, Batsakis JG. Carcinomas ex pleomorphic adenoma and malignant mixed tumors. Histomorphologic indexes. Arch Otolaryngol 1984;110:172-6.

8 Chen KT, Weinberg RA, Moseley D. Carcinosarcoma of the salivary gland. Am $\mathcal{F}$ Otolaryngol 1984;5:415-17.

9 Yamamoto Y, Kishimoto Y, Virmani AK, et al. Mutations associated with carcinomas arising from pleomorphic adenomas of the salivary glands. Hum Pathol 1996;27:7826.

10 Kamio N. Coexpression of p53 and c-erbB-2 proteins is associated with histological type, tumour stage and cell proliferation in malignant salivary gland tumours. Virchows Arch 1996;428:75-83. 